\title{
Phases of anisotropic dipolar antiferromagnets: Mean field theory and Monte Carlo simulations
}

\author{
Julio F. Fernández ${ }^{1, *}$ and Juan J. Alonso ${ }^{2, \dagger}$ \\ ${ }^{1}$ ICMA, CSIC, and Universidad de Zaragoza, 50009-Zaragoza, Spain \\ ${ }^{2}$ Física Aplicada I, Universidad de Málaga, 29071-Málaga, Spain
}

(Received 1 October 2005; revised manuscript received 8 December 2005; published 19 January 2006)

\begin{abstract}
We study systems of classical magnetic dipoles on simple cubic lattices with dipolar and antiferromagnetic exchange interactions. By analysis and Monte Carlo (MC) simulations, we find how the antiferromagnetic phases vary with uniaxial and fourfold anisotropy constants ( $C$ and $D$ respectively), as well as with exchange strength $J$. We pay special attention to the spin reorientation (SR) phase, and exhibit in detail the nature of its broken symmetries. By mean field theory and by MC, we also obtain the ratio of the higher ordering temperature to the SR transition temperature, and show that it depends mainly on $D / C$, and rather weakly on $J$. We find a reverse SR transition.
\end{abstract}

DOI: 10.1103/PhysRevB.73.024412

\section{INTRODUCTION}

Long-range magnetic order brought about by purely dipolar interactions is somewhat rare in nature. ${ }^{1}$ Interest in the subject is nevertheless growing. Some of it comes from the availability of synthesized crystals of organometallic molecules $^{2}$ that behave at low temperature as single spins. ${ }^{3-6}$ Owing to the large organic mass enveloping the magnetic cores of these molecules, dipole-dipole interactions are then dominant. ${ }^{7}$ Long-range order has already been observed experimentally. ${ }^{8-12}$ Because uniaxial anisotropy is very large, Ising spins with dipolar interactions are reasonable models for these systems. ${ }^{13}$ Early rigorous work by Luttinger and Tisza $^{14}$ established which type of magnetic order obtains at low temperature $T$ in dipolar Ising models in each of the cubic lattices. The same results have been arrived at more recently by simpler methods. ${ }^{15}$

No Ising model can, however, account for some of the interesting collective behavior, such as spin reorientation ${ }^{16-20}$ (SR) and canted (i.e., noncollinear) spin configurations, which can be induced by purely dipolar interactions. Accordingly, three component spin models that include exchange as well as dipolar interactions are often used when modeling these effects. ${ }^{21,22}$ To explore the transition between the dipolar- and exchange-dominated antiferromagnetic phases is one of the aims of this paper.

The SR transition deserves special attention. Thermally driven SR transitions take place well within an ordered magnetic phase. All spins rotate as a whole as the temperature decreases below some SR transition temperature $T_{r}$. SR transitions can be continuous, as the ones first observed in the bulk ${ }^{16,17}$ or first order, as often observed in films. ${ }^{18-20}$ It is important to realize that a SR phase comes with continuous transitions. ${ }^{23}$ This phase is defined by its own unique set of broken symmetries. One of the aims of this paper is to exhibit this in detail.

It has long been realized that higher-order anisotropies are required for the existence of the SR phase. ${ }^{17,23,24}$ Accordingly, we choose to study the model Hamiltonian

$$
\mathcal{H}=\mathcal{H}_{J}+\mathcal{H}_{d}+\mathcal{H}_{A},
$$

where $\mathcal{H}_{J}$ and $\mathcal{H}_{d}$ are for all exchange and dipolar interactions, respectively,

$$
\mathcal{H}_{A}=-D \sum_{i}\left(S_{i}^{z}\right)^{2}-C \sum_{i}\left[\left(S_{i}^{x}\right)^{4}+\left(S_{i}^{y}\right)^{4}\right]
$$

$D$ and $C$ are constants, and $S_{i}^{\alpha}$ is the $\alpha$ component ( $\alpha$ $=x, y, z)$ of a unit spin $S$ at site $i$.

The fourfold anisotropy in Eq. (2) contributes the desired competition to the uniaxial anisotropy. Such a term arises naturally in tetragonal crystal lattices, and will be seen to bring about the canted spin phase that is shown in the lower left quadrant of Fig. 1.

To continue with the definition of the model, we let

$$
\mathcal{H}_{J}=-J \sum_{\langle i j\rangle} \mathbf{S}_{i} \cdot \mathbf{S}_{j},
$$

where $\Sigma_{\langle i j\rangle}$ is a sum over all nearest-neighbor bonds, and $\mathbf{S}_{i}$ is a classical three-component unit spin at lattice site $i$. We also consider nearest-neighbor exchange interactions in order to establish how small $|J|$ must be for dipolar magnetic order to obtain.

Finally, $\mathcal{H}_{d}=(1 / 2) \Sigma_{i j} \Sigma_{\alpha \beta} T_{i j}^{\alpha \beta} S_{i}^{\alpha} S_{j}^{\beta}$, where the sum is here over all sites $i$ and $j$ except $i=j$,

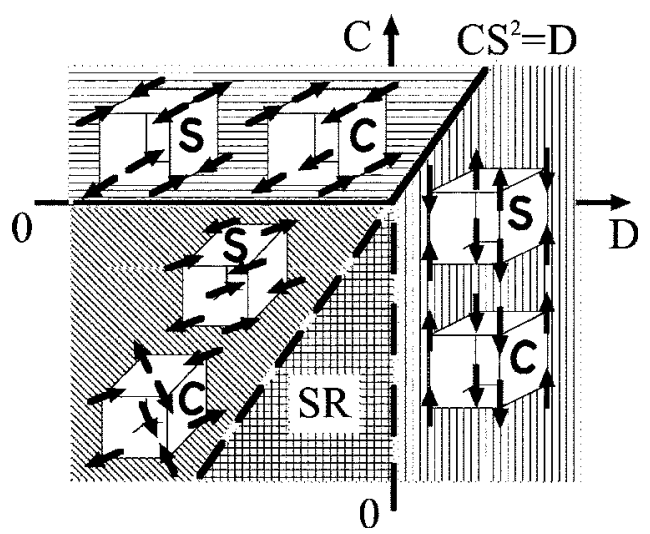

FIG. 1. Phase diagram for $T=0$. The types of orders labeled $\mathfrak{c}$ and $\mathfrak{s}$ are for $-1.34 \varepsilon_{d} \leq J<0$ and $J \lesssim-1.34 \varepsilon_{d}$, respectively. Full and dashed thick lines stand for first- and second-order transitions, respectively. 


$$
T_{i j}^{\alpha \beta}=\varepsilon_{d}\left(\frac{a}{r_{i j}}\right)^{3}\left(\delta_{\alpha \beta}-3 \frac{r_{i j}^{\alpha} r_{i j}^{\beta}}{r_{i j}^{2}}\right),
$$

and $\mathbf{r}_{i j}$ is the displacement between sites $i$ and $j$. We let $E_{A}$, $E_{J}$, and $E_{d}$ be the values of $\mathcal{H}_{A}, \mathcal{H}_{J}$, and $\mathcal{H}_{d}$, respectively, for whatever state is specified.

Since we treat all spins as classical unit vectors, no quantum effect is taken into account. Accordingly, we disregard hyperfine interactions, since they have no effect on the thermal equilibrium behavior of electronic spins when the latter are treated classically. ${ }^{25}$ Quantum effects, such as the enhancement of the transverse field that is necessary in order to destroy long-range order in $\mathrm{LiHoF}_{4}$ below approximately $1 \mathrm{~K},{ }^{26}$ are therefore beyond the scope of this paper. Thus, some numbers we obtain, such as the crossover value of $J$, below which dipolar interactions become dominant, may change a little when hyperfine interactions are included in a quantum treatment. On the other hand, our main results, i.e., the insensitivity to the value of $J$ to the ratio of the ordering temperature to the SR temperature, as well as the existence of a reverse SR transition, are of a rather qualitative nature, and are therefore expected to be insensitive to quantum effects.

Simple cubic (sc) lattices and zero applied magnetic field $H$ are assumed throughout. We only work with $L \times L \times L$ box-like systems, and let dipole-dipole interactions act between each spin and all other spins within an $L \times L \times L$ box centered on it. We use periodic boundary conditions, because they give faster convergence towards the $L \rightarrow \infty$ limit than free boundary conditions do. In addition, because we work with antiferromagnets, and not ferromagnets, convergence is faster than it would otherwise be. Most of our results follow from simulations for $L=8$ and $L=16$. This would be insufficient for critical behavior work but is adequate for our purposes; that is, establishing which type of magnetic order obtains and the corresponding phase boundaries. It is worth recalling that thermal equilibrium results obtained for $H=0$ for large cubic-shaped systems can, by virtue of Griffith's theorem, ${ }^{28}$ be generalized to other shapes in three dimensions (3D).

Our simulations follow the standard Metropolis Monte Carlo (MC) algorithm. ${ }^{27}$ More specifically, we start simulations with an initial configuration in which all spins point in either random or parallel directions. We next compute the dipolar field at each site. Time evolution takes place as follows. A spin is chosen at random and temporarily pointed in a new random direction. Let $\Delta E$ be the corresponding energy change. If $\Delta E<0$, the temporary direction becomes permanent. If, on the other hand, $\Delta E>0$, the temporary direction becomes permanent only with probability $\exp \left(-\Delta E / k_{B} T\right)$, where $T$ is the system's temperature. The field changes that ensue at every site in the system if the new spin direction is accepted are then computed, thus updating the field values everywhere on the system.

The plan of the paper and a list of the results obtained follow. In Sec. II, we study all the phases, except the SR phase (see Fig. 1), for all $J \leqslant 0$. To this end, we first define a canted state that lets all spins point along the easy magnetization axes for any $C$ and $D$, while $E_{d}$ remains invariant if the system's shape is cubic. This is the main device that enables us to conclude in Sec. II that $\mathfrak{c}$ and $\mathfrak{s}$, exhibited in Fig. 1, are shown to be the ground state configurations for $-1.34 \varepsilon_{d}<J<0$ and $J<-1.34 \varepsilon_{d}$, respectively. We also report $\mathrm{MC}$ data which suggest that, as in the Landau theory, ${ }^{23}$ the $x y$-collinear to $x y$-canted transition line is first order. We also report results from $\mathrm{MC}$ simulations for temperature-driven phase transitions between paramagnetic and $x y$-collinear and between $x y$-collinear and $x y$-canted phases. In Sec. III we study the SR phase. In an anisotropy diagram we show the free energy minima that follow from MC simulations for the SR phase. The broken symmetries are clearly exhibited. This suggests how to write mean field equations for the SR transition, from which we obtain $T_{r}$ as well as the higher ordering temperature as a function of $D / C$. We show that the ratio of these two temperatures depends mainly on the $D / C$ ratio and rather weakly on $J$, and that mean field yields approximately the same value for it as MC simulations do. For $D$ $<0$ and $0.8 \leqq D / C<1$, cooling through the paramagnetic phase first brings the system into $x y$-canted ordering. The SR phase is encountered at a lower temperature. For $D<0$ but $0<D / C \leqq 0.8$, cooling through the paramagnetic phase first brings the system into z-collinear ordering, and the SR phase is encountered at a lower temperature. An interesting phase diagram obtains at $D / C \sim 0.8$, and a reverse spin reorientation that is temperature driven is observed. A complete spin reorientation, from the easy magnetization axis into the perpendicular plane, first takes place upon cooling below the paramagnetic phase, followed, upon further cooling, by a reverse spin reorientation towards the easy magnetization axis. This is so for $J=0$ as well as for $\varepsilon_{d}=0$.

\section{COLLINEAR AND CANTED PHASES}

In this section we study all the phases shown in Fig. 1, except the SR phase. We first study how the ground state of a purely dipolar system varies with $D$ and $C$. We then extend this to all $J<0$, and finally consider nonzero temperatures.

\section{A. The canted state}

In this subsection we define the canted state $\mathfrak{c}$. We do this in order to avoid the difficulty that follows from the the fact that dipolar interactions are not rotationally invariant, thus making the task of simultaneously minimizing $E_{A}$ and $E_{d}$ nontrivial. The canted state $c$ enables one to do this minimization. Let

$$
S_{i}^{z}=\tau_{i}^{z} \cos \theta, S_{i}^{y}=\tau_{i}^{y} \sin \theta \sin \phi, S_{i}^{x}=\tau_{i}^{x} \sin \theta \cos \phi,
$$

where $\theta$ is the angle between the spin vector and the $z$ axis, $\phi$ is the azimuthal angle, $\tau_{i} \equiv\left[\tau_{i}^{x}, \tau_{i}^{y}, \tau_{i}^{z}\right]$ is given by

$$
\boldsymbol{\tau}_{i}=\left[(-1)^{y(i)+z(i)},(-1)^{x(i)+z(i)},(-1)^{x(i)+y(i)}\right],
$$

$[x(i), y(i), z(i)]$ is the position of site $i$ and $x(i), y(i)$, and $z(i)$ are all integers.

Before proceeding with the argument, we point out some basic features of the canted state we have just defined. Note how $\tau_{i}$ varies with $i$. A canted spin configuration therefore follows from Eqs. (5) and (6) if $\theta \neq 0$ and $\theta \neq \pi / 2$. For $\theta$ 
$=\pi / 2$, a canted spin configuration also obtains if $\phi \neq 0$ and $\phi \neq \pi / 2$, as, for instance, the $\mathfrak{c}$ state in the lower left-hand quadrant of Fig. 1. The fact that our definition of a $\mathfrak{c}$ state gives a collinear spin configuration for these few special cases (see the $\mathfrak{c}$ state in the lower right-hand corner of Fig. 1 for $\theta=0$ ) should not be too confusing.

To start our argument, note that $\theta=0$ gives the minimum value of $E_{d}$ for sc lattices in the bulk. ${ }^{14,28}$ It is next shown that the dipolar energy $E_{d}$ of cubic-shaped systems is independent of $\theta$ and of $\phi$ in the canted state. ${ }^{29}$ In order to see this, consider first $\mathbf{S}_{i} \cdot \mathbf{S}_{j}$, which upon substitution of Eq. (5) becomes $\tau_{i}^{z} \tau_{j}^{z} \cos ^{2} \theta+\tau_{i}^{y} \tau_{j}^{y} \sin ^{2} \theta \sin ^{2} \phi+\tau_{i}^{x} \tau_{j}^{x} \sin ^{2} \theta \cos ^{2} \phi$. Now it is easy to check that the sum $\Sigma_{i, j} \mathbf{S}_{i} \cdot \mathbf{S}_{j} f\left(r_{i j}\right)$, where $f\left(r_{i j}\right)$ is any function of $r_{i j}$, over a sc lattice bounded by the surface of a cube is independent of $\theta$ and of $\phi$, since $\sum_{i, j} \tau_{i}^{z} \tau_{j}^{z} f\left(r_{i j}\right)=\sum_{i j} \tau_{i}^{y} \tau_{j}^{y} f\left(r_{i j}\right)=\sum_{i j} \tau_{i}^{x} \tau_{j}^{x} f\left(r_{i j}\right)$. Thus, the first of the two terms of the dipolar interaction is independent of $\theta$ and of $\phi$, and, by the way, so is the exchange energy. Now, consider $w_{i j} \equiv\left(x_{i j} S_{i}^{x}+y_{i j} S_{i}^{y}+z_{i j} S_{i}^{z}\right)\left(x_{i j} S_{j}^{x}+y_{i j} S_{j}^{y}+z_{i j} S_{j}^{z}\right)$, from the second term of the dipolar interaction. Again, $\Sigma_{i j} w_{i j} / r_{i j}^{3}$ over a sc lattice bounded by the surface of a cube is independent of $\theta$ and of $\phi$, since (1) cross-terms do not contribute to the sum, by reflection symmetry, and (2) $\Sigma_{i, j} \tau_{i}^{z} \tau_{j}^{z} / r_{i j}^{3}$ $=\Sigma_{i j} \tau_{i}^{y} \tau_{j}^{y} / r_{i j}^{3}=\Sigma_{i j} \tau_{i}^{x} \tau_{j}^{x} / r_{i j}^{3}$. Therefore, $\theta$ and $\phi$ can be freely chosen in Eq. (5) in order to minimize $E_{A}$, at no cost to $E_{d}$, which is the desired result for bulk systems.

Finally, since the minimum value of $E_{A}$ can be reached by appropriate choice of $\theta$ and of $\phi$ in Eq. (5), it follows that minimization of $E_{A}$ with the $\mathfrak{c}$ state gives the ground state.

The phases obtained for the ground state by this procedure if $J=0$ are shown in Fig. 1. We refer to the states on the right-hand side, upper left-hand side and lower left-hand sides of the diagram as $z$-collinear, $x y$-collinear, and $x y$-canted phases, respectively. The nature of the SR phase is the subject of Sec. III.

Note that the $x y$-collinear phase is unstable with respect to any nonvanishingly small $C<0$. [On the other hand (see below), the $x y$-canted phase obtains in finite nonzero temperature only for a sufficiently small $C<0$.]

Before we proceed any further, we need some additional definitions. Let

$$
m_{\mathfrak{c}}^{\alpha}=N^{-1} \sum_{i} S_{i}^{\alpha} \tau_{i}^{\alpha}
$$

In a $\mathfrak{c}$ state, $m_{\mathrm{c}}^{z}=\cos \theta, m_{\mathrm{c}}^{x}=\sin \theta \cos \phi$, and $m_{\mathrm{c}}^{y}=\sin \theta \sin \phi$.

According to Landau's theory, the transition between the $z$-collinear and the $x y$-plane phases, as well as the transition between the $x y$-collinear and the $x y$-canted phases, is of first order. ${ }^{23}$ This has been observed for the $z$-collinear to $x y$-plane transition in films. ${ }^{19,22,30}$ The results we have obtained from MC simulations also support this conclusion.

On the other hand, we know of no experimental or MC evidence about the nature of the $x y$-collinear to $x y$-canted transition. Data points from MC simulations we have performed are plotted in Fig. 2. Note: (1) $m_{\mathfrak{c}}^{z}=0$ for all -1 $<C / \varepsilon_{d}<1$, (2) $m_{\mathrm{c}}^{x}=m_{\mathrm{c}}^{y}$ for all $C / \varepsilon_{d}<0$, and (3) either (a) $m_{\mathrm{c}}^{x}=0$ and $m_{\mathrm{c}}^{y}=1$ or (b) $m_{\mathrm{c}}^{x}=1$ and $m_{\mathrm{c}}^{y}=0$ for $C>0$. A firstorder phase transition between two in-plane spin configura-

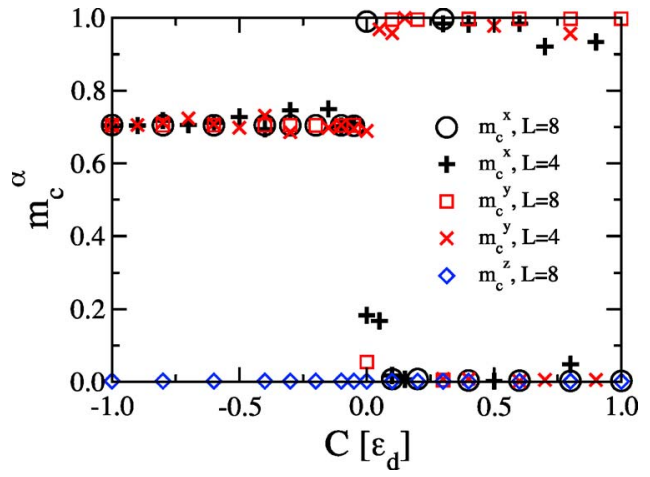

FIG. 2. (Color online) Order parameter $m_{\mathrm{c}}^{\alpha}$, for $\alpha=x, y, z$, vs $C$ for systems of $L \times L \times L$ dipoles on sc lattices at $T=0.04 \varepsilon_{d} / k_{B}$, where $k_{B}$ is Boltzmann's constant, for $D=-3 \varepsilon_{d}$. A transition between the $x y$-collinear and $x y$-canted phases is clearly exhibited at $C=0$. In order to reach equilibrium, $T$ was lowered from $T$ $=2 \varepsilon_{d} / k_{B}$ in $\Delta T=-0.02 \varepsilon_{d} / k_{B}$ steps. $2 \times 10^{4} \mathrm{MC}$ sweeps were made at each temperature step. Equilibrium values come from averages over $2 \times 10^{5}$ MC sweeps.

tions, one along the $x$ or $y$ axes and the other one with $\phi$ $=\pi / 2$, is clearly suggeted.

\section{B. Exchange interaction}

The effect exchange interactions have on the ground state is studied in this subsection.

Let us first define the collinear $\mathfrak{s}$ state, illustrated in Fig. 1 by one of the two sets of the states. In this state, all nearestneighbor spins to spin $\mathbf{S}$ point opposite to $\mathbf{S}$ (as in Fig. 1). Alternatively, in an $\mathfrak{s}$ state,

$$
S_{i}^{z}=\eta_{i} \cos \theta, S_{i}^{y}=\eta_{i} \sin \theta \sin \phi, S_{i}^{x}=\eta_{i} \sin \theta \cos \phi,
$$

where

$$
\eta_{i} \equiv(-1)^{x(i)+y(i)+z(i)} .
$$

It makes sense to also define

$$
m_{\mathfrak{s}}^{\alpha}=N^{-1} \sum_{i} S_{i}^{\alpha} \eta_{i}
$$

In an $\mathfrak{s} \quad$ state, $\quad m_{\mathfrak{s}}^{z}=\cos \theta, \quad m_{\mathfrak{s}}^{x}=\sin \theta \cos \phi, \quad$ and $\quad m_{\mathfrak{s}}^{y}$ $=\sin \theta \sin \phi$, but in a c state, $\mathbf{m}_{\mathfrak{s}}=0$.

We showed in Sec. II A that $\mathfrak{c}$ is the ground state if $J=0$ and that $E_{d}$ is independent of $\theta$ and $\phi$ in Eq. (5). In addition, $E_{d}=-2.67 \varepsilon_{d}{ }^{15}$ Note also that Eqs. (5) and (6) imply $E_{J}=J$ for all $\theta$ and $\phi$ in a c state. Similarly, it can be easily checked that in an $\mathfrak{s}$ state, $E_{d}=0$, independent of the direction of $\mathbf{S}$, and that $E_{J}=-3 J$.

Therefore, of the above two states, the $\mathfrak{c}$ state gives the lower energy in the $-1.34 \varepsilon_{d}<J<0$ range and the $\mathfrak{s}$ state gives the lower energy in the $J<-1.34 \varepsilon_{d}$ range. Monte Carlo simulations show that no other state gives a lower energy in the whole $J<0$ range, that is,

$$
E=-2.67 \varepsilon_{d}+J+E_{A}
$$

if $-1.34 \varepsilon_{d} \leq J<0$ and 


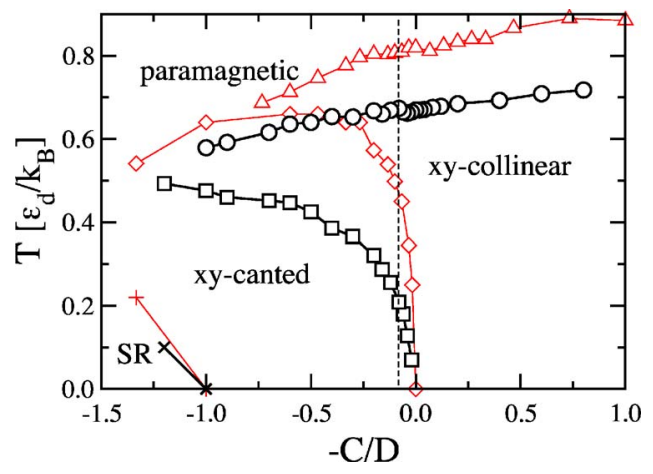

FIG. 3. (Color online) Transition temperatures and phases for systems with only dipolar interactions $(J=0)$, and two values of $D$ : $-3 \varepsilon_{d}(\triangle$ and $\diamond)$, and $-0.5 \varepsilon_{d}(\bigcirc$ and $\square)$. + are for the SR phase boundary. The data come from MC simulations of systems of 8 $\times 8 \times 8$ spins on sc lattices. The vertical dashed line stands for the cooling path that was taken to obtain the data points shown in Fig. 4.

$$
E=3 J+E_{A}
$$

if $J<-1.34 \varepsilon_{d}$.

Clearly, $\partial E / \partial J$ is discontinuous at $J \simeq-1.34 \varepsilon_{d}$, implying, a first-order transition between purely dipolar induced canted (i.e., a c state) and collinear antiferromagnetic phases (i.e., an $\mathfrak{s}$ state) for all $C$ and $D$.

\section{C. $T \neq 0$}

Of all thermally driven transitions between any two of the paramagnetic, $x y$-collinear, and $x y$-canted phases (the SR phase is treated in Sec. III), the most interesting one is the one between the $x y$-collinear and the $x y$-canted phases. It is illustrated in Figs. 3 and 4. This effect has been discussed before for thin films. ${ }^{31}$ Note how the variation of $\mid\left[m_{\mathfrak{c}}^{x}\right]^{2}$ $-\left[m_{\mathfrak{c}}^{y}\right]^{2} \mid$ and of $\left|m_{\mathfrak{c}}^{x} m_{\mathfrak{c}}^{y}\right|$ at $T \simeq 0.24 \varepsilon_{d}$ becomes sharper as the

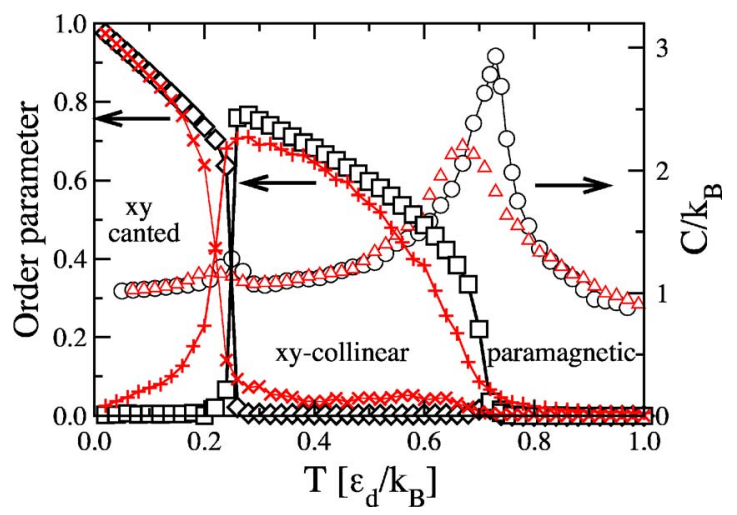

FIG. 4. (Color online) $\left|\left[m_{\mathfrak{c}}^{x}\right]^{2}-\left[m_{\mathfrak{c}}^{y}\right]^{2}\right|\left[2 m_{\mathfrak{c}}^{x} m_{\mathfrak{c}}^{y}\right]^{2}$ and $C / k_{B}$ vs $T$ for systems of $L \times L \times L$ spins with $J=0$, and $D=-0.5 \varepsilon_{d}$, and $C$ $=0.05 D$. For $L=8,+($ red online $), \times$ (red online), and $\triangle$ (red online) stand for $\left|\left[m_{\mathrm{c}}^{x}\right]^{2}-\left[m_{\mathrm{c}}^{y}\right]^{2}\right|,\left[2 m_{\mathrm{c}}^{x} m_{\mathrm{c}}^{y}\right]^{2}$, and $C / k_{B}$, respectively; for $L=16, \square, \diamond$, and $\bigcirc$ stand for $\left|\left[m_{\mathfrak{c}}^{x}\right]^{2}-\left[m_{\mathfrak{c}}^{y}\right]^{2}\right|,\left[2 m_{\mathfrak{c}}^{x} m_{\mathfrak{c}}^{y}\right]^{2}$, and $C / k_{B}$, respectively. For $L=16(L=8)$, all data points stand for averages over $10^{5}\left(2.5 \times 10^{5}\right) \mathrm{MC}$ sweeps.

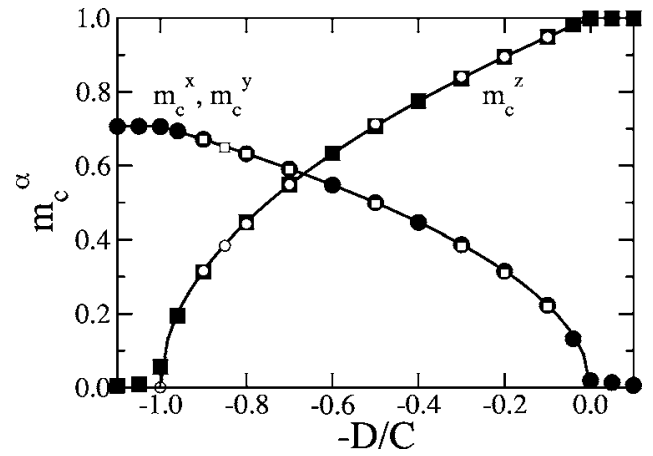

FIG. 5. $m_{\mathrm{c}}^{\alpha}$ vs $-D / C$ for $\alpha=x, y, z$ and $T=0$. stands for both $m_{\mathfrak{c}}^{x}$ and $m_{\mathfrak{c}}^{y}$, and $\square$ stands for $m_{\mathfrak{c}}^{z}$, for $C=-0.5 \varepsilon_{d}$ and $J=0$; $\square$ stands for both $m_{\mathfrak{c}}^{x}$ and $m_{\mathfrak{c}}^{y}$, and $\bigcirc$ stands for $m_{\mathfrak{c}}^{z}$, for $\varepsilon_{d}=0$ and $C=J<0$. All symbols are from MC simulations in which $T$ was lowered from $T \gg \varepsilon_{d},|J|$ to $T \ll \varepsilon_{d},|J|$. Continuous lines are from Eq. (13) and below.

systems size increses. This suggests a first-order phase transition, in accordance with Landau's theory. ${ }^{23}$

\section{THE SPIN REORIENTATION PHASE}

\section{A. $T=0$}

We first explore $\mathrm{SR}$ at $T=0$. We start by minimizing the anisotropy energy $E_{A}$ for all $C$ and $D$ in the SR phase (see Fig. 1). Recall from Sec. II that $E_{A}$ can be minimized freely for all $J \leqslant 0$. We obtain

$$
\mathbf{S}=( \pm u, \pm u, \pm v)
$$

for $C<D<0$, where $u=\sqrt{D / 2 C}$ and $v=\sqrt{1-2 u^{2}}$. Thus, varying $D$ through the $C<D<0$ range forces the spin directions to vary in the ground state from the $z$-collinear state shown in Fig. 1 to the $x y$-phase. This is analogous to the phenomenological theory of SR. ${ }^{23}$

We have simulated cooling from high temperatures down to $k_{B} T \ll \varepsilon_{d}, J$ for various values of $C$ and $D$ : (1) purely dipolar interacting 3D systems (i.e., $J=0$ ) and (2) antiferromagnetic 3D systems with only nearest-neighbor interactions (i.e., $J<0, \varepsilon_{d}=0$ ). The MC results obtained as well as the numbers that follow from Eq. (13) are plotted in Fig. 5. The data points fall on the predicted curves, independently of $J$.

Note that Eq. (13) allows eight different spin directions. This suggests which symmetries are broken in the SR phase. We examine this idea in more detail in the following section.

\section{B. The temperature-driven transition}

In Fig. $6, m_{\mathfrak{c}}^{\alpha}$ for $\alpha=x, y, z$, and the specific heat $C$ are plotted vs $T$ for $J=0$, and $D=-0.5 \varepsilon_{d}, C=-\varepsilon_{d}$. The data points are from MC simulated cooling. Data points obtained from heating from ordered states, using Eq. (13) and below, do not differ from the ones shown.

Specific-heat curves obtained for various system sizes are shown in Fig. 7. The data are not good enough for an accurate value of any critical index, but they suggest the transitions are continuous at both of the two transition temperatures. 


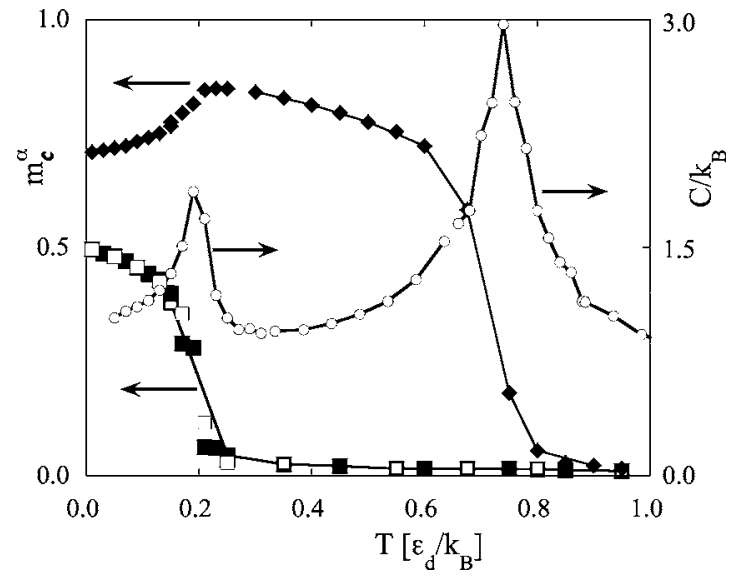

FIG. 6. $m_{\mathfrak{c}}^{z}(\bullet), m_{\mathfrak{c}}^{y}(\square), m_{\mathfrak{c}}^{x}(\square)$, and $C / k_{B}(\bigcirc)$ vs $T$. All data points come from MC simulations of $8 \times 8 \times 8$ spins on sc lattices for $J=0, C=-\varepsilon_{d}$, and $D=0.5 C$. At each value of $T$, at least $10^{5} \mathrm{MC}$ sweeps were made. Lines are guides to the eye.

We have obtained additional specific heat and $\mathbf{m}_{\mathfrak{c}}$ curves (as the ones in Fig. 6) for $J=0$ and $C=-3 \varepsilon_{d}$, from further MC simulations. The data points shown in Fig. 8 for the two transition temperatures as well as the labels shown for the phases follow from such curves.

For all $J<0$, the phase diagram shown in Fig. 8 is qualitatively the same, except that for $J<-1.3 \varepsilon_{d}$, the $x y$-canted phase is replaced by the collinear $x y$ phase shown in Fig. 1 for $C, D<0$.

\section{Broken symmetries}

In this section, we illustrate the symmetries of the various magnetic phases a system goes through when cooling from the paramagnetic into the SR phase. To this effect, a statisti-

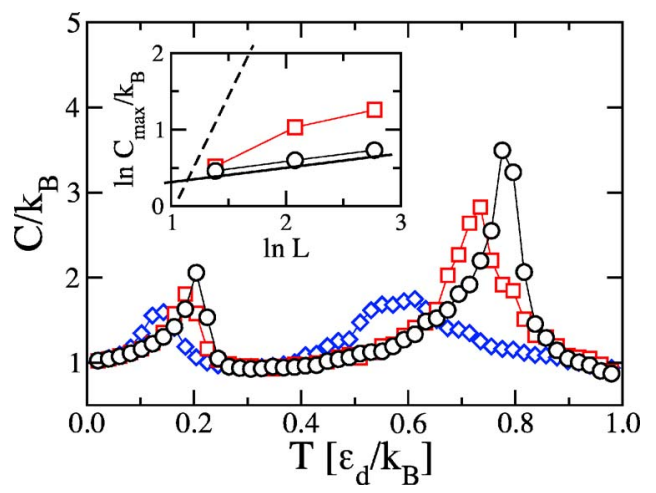

FIG. 7. (Color online) Specific heat vs temperature for systems of $L \times L \times L$ dipoles, with $J=0, C=-\varepsilon_{d}$ and $D=0.5 C$. $\bigcirc, \square$, and $\diamond$ stand for $L=16,8$, and 4 respectively. $T$ was lowered in $\Delta T=$ -0.01 steps. At each value of $T$, we made $5 \times 10^{4}, 5 \times 10^{5}$ and 2 $\times 10^{7} \mathrm{MC}$ sweeps for $L=16,8$, and 4, respectively. The high (low) temperature peak corresponds to the paramagnetic to $z$-collinear phase (z-collinear to SR phase) transition. Inset: $\log C_{\max } / k_{B}$ vs $\log L$ for both transitions. $\bigcirc(\square)$ stands for the high (low) $T$ peaks. The slope of the dashed line is for a first-order transition $(C \sim N)$. The slope of the full line is 0.2 , in accordance with a countinuous transition in which $\alpha / \nu \approx 0.2$.

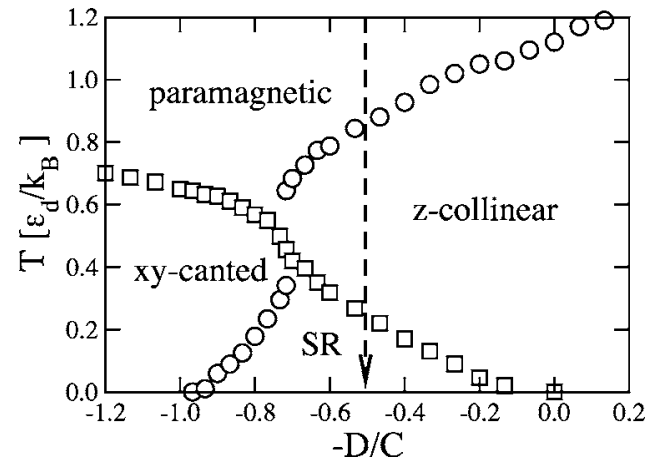

FIG. 8. (a) Phases of dipolar $(J=0)$ antiferromagnets for $C=$ $-3 \varepsilon_{d}$. All data points come from MC simulations of $8 \times 8 \times 8$ spins. The dashed line is for the cooling path from which the data points in Fig. 6 follow.

cal sample of values $\mathbf{m}_{\mathfrak{c}}$ takes up throughout time in each of the three phases-paramagnetic, $z$-collinear, and $S R$-that obtain for $C=-\varepsilon_{d}$ and $D=0.5 C$ is shown in Figs. 9(a) and 9(b).

Now,

$$
p\left(\mathbf{m}_{\mathfrak{c}}\right)=Z^{-1} e^{-F\left(\mathbf{m}_{\mathfrak{c}}\right) / k_{B} T},
$$

where $p\left(\mathbf{m}_{\mathfrak{c}}\right)$ is the probability to find a $\mathbf{m}_{\mathfrak{c}}$ value and $F\left(\mathbf{m}_{\mathfrak{c}}\right)$ is the free energy. It follows that cloud densities in Figs. 9(a) and 9 (b) are proportional to $\exp \left[-F\left(\mathbf{m}_{\mathfrak{c}}\right) / k_{B} T\right]$. The clouds, therefore, stand for neighborhoods of the minima of $F\left(\mathbf{m}_{\mathfrak{c}}\right)$ for each of the three phases.

The data points shown in Fig. 9(a) for each value of $T$ come from one single $\mathrm{MC}$ run. This is in contrast to the procedure used to obtain the data points shown in Fig. 9(b), which were obtained from $10^{3}$ independent $\mathrm{MC}$ runs of 4 $\times 10^{4} \mathrm{MC}$ sweeps each.

Clearly, all the symmetries that are broken in the $x y$-canted phase, in addition to the symmetries that are broken in the $z$-collinear phase, are broken in the SR phase.

\section{Mean field}

It is interesting to calculate the mean field transition temperatures associated with the SR phase. We assume a magnetic order as either in a canted or a collinear state, and that only $\mathbf{m}_{\mathfrak{c}}$ and $\mathbf{m}_{\mathfrak{s}}$ remain to be determined. More precisely, we assume

$$
\left\langle S_{i}^{\alpha}\right\rangle=m_{\mathfrak{c}}^{\alpha} \tau_{i}^{\alpha}+m_{\mathfrak{s}}^{\alpha} \eta_{i} .
$$

We now write the mean field equations. First note that multiplying the above equation by $\tau_{i}^{\alpha}$ and summing over two nearest-neighbor sites along the $\alpha$ direction gives

$$
2 m_{\mathfrak{c}}^{\alpha}=\sum_{\alpha, i=1,2} \tau_{i}^{\alpha}\left\langle S_{i}^{\alpha}\right\rangle .
$$

Similarly, one obtains

$$
2 m_{\mathfrak{s}}^{\alpha}=\sum_{\alpha, i=1,2} \eta_{i}\left\langle S_{i}^{\alpha}\right\rangle
$$

We now write the main mean field equation 
(a)

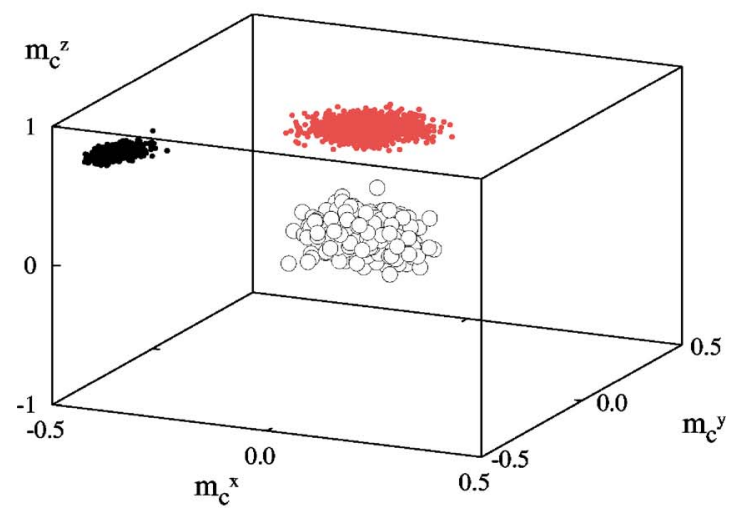

(b)

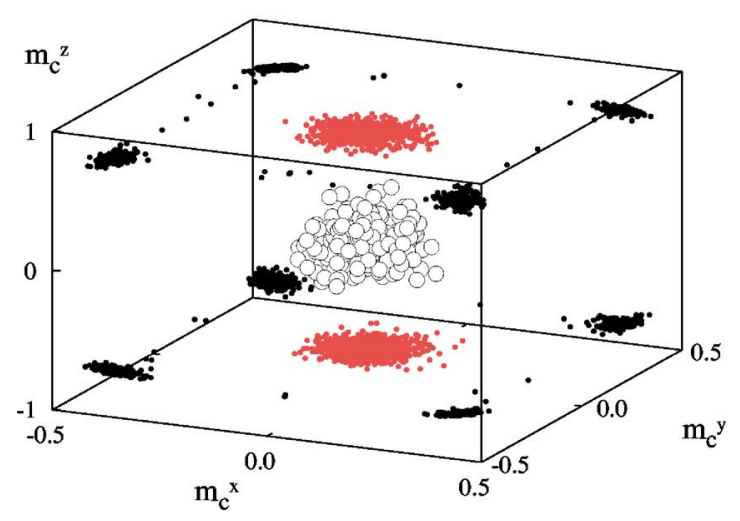

FIG. 9. (Color online) (a) $\mathbf{m}_{\mathfrak{c}}$ values measured every $10^{3}$ equally spaced MC sweeps in equilibrium for systems of $8 \times 8 \times 8$ dipoles with $J=0, C=-\varepsilon_{d}$, and $D=0.5 C$. Each set of $\bigcirc$, gray (red online), and black dots stand for $k_{B} T / \varepsilon_{d}=0.9,0.5$, and 0.15 , respectively. All data points for each $T$ come from a single MC run of $4 \times 10^{6}$ MC sweeps each. In each MC run, before we take any data, we let the system equilibrate by lowering the temperature from $T$ $=2 \varepsilon_{d} / k_{B}$ (deep in the paramagnetic phase) in steps of $\Delta T=-0.1$, of $4 \times 10^{3}$ MC sweeps each. ( b) Same as in a) but each of the three sets of points comes from $10^{3}$ independent short $\mathrm{MC}$ runs (i.e., first cooling from $T=2 \varepsilon_{d} / k_{B}$ in each of the $10^{3} \mathrm{MC}$ runs) of $4 \times 10^{4} \mathrm{MC}$ sweeps steps each.

$$
\left\langle\mathbf{S}_{i}\right\rangle=Z^{-1} \sum_{\mathbf{u}} \mathbf{u} \exp \left[-\varepsilon_{i}(\mathbf{u}) / k_{B} T\right]
$$

where the sum is over all directions of the unit vector $\mathbf{u}$,

$$
\begin{gathered}
\varepsilon_{i}(\mathbf{u})=-\mathbf{h}(i) \cdot \mathbf{u}+\varepsilon_{A}(\mathbf{u}), \\
\varepsilon_{A}(\mathbf{u})=-D u_{z}^{2}-C\left(u_{x}^{4}+u_{y}^{4}\right),
\end{gathered}
$$

$\mathbf{h}(i)=\mathbf{h}_{J}(i)+\mathbf{h}_{d}(i)$,

$$
h_{J}^{\alpha}(i)=J\left(m_{\mathfrak{c}}^{\alpha} \sum_{n i} \tau_{i}^{\alpha}+m_{\mathfrak{s}}^{\alpha} \sum_{n i} \eta_{i}\right)
$$

the sum here is over all nearest neighbors of site $i$,

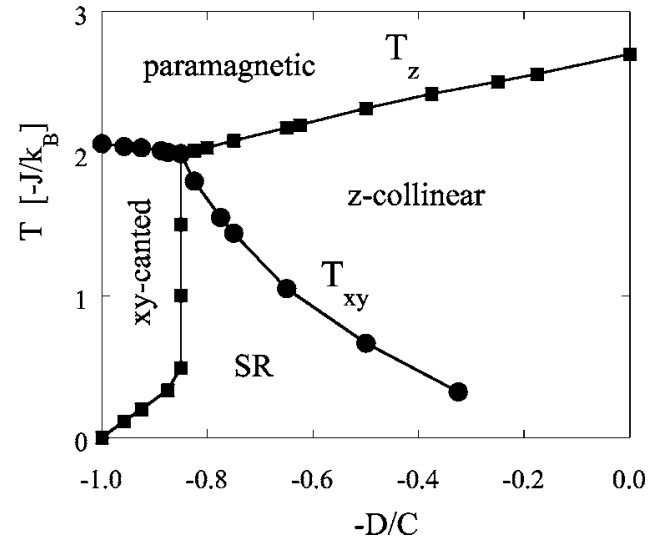

FIG. 10. Mean field transition temperatures $T_{z}$ and $T_{x y}$ and the nature of the antiferromagnetic phases vs $-D / C$ for $3 \mathrm{D}$, nearestneighbor interactions only (i.e., $\varepsilon_{d}=0$ ), $J<0$, and $C=4 J$.

$$
h_{d}^{\alpha}(i)=\sum_{j} \sum_{\beta} T_{i j}^{\alpha \beta}\left(m_{\mathfrak{c}}^{\beta} \tau_{j}^{\beta}+m_{\mathfrak{s}}^{\beta} \eta_{j}\right)
$$

and, finally,

$$
Z=\sum_{\mathbf{u}} \exp \left[-\varepsilon_{i}(\mathbf{u}) / k_{B} T\right]
$$

The above equations simplify near the phase boundaries. Consider, for instance, the boundary between the paramagnetic and the $z$-collinear phases in Fig. 10. Let $T_{z}$ define the boundary between a phase where $m_{\mathfrak{c}}^{z}$ and $m_{\mathfrak{s}}^{z}$ vanish and a phase where either $m_{\mathfrak{c}}^{z}$ or $m_{\mathfrak{s}}^{z}$ does not. $T_{x y}$ is similarly defined for the $x$ and $y$ components of $\mathbf{m}_{\mathfrak{s}}$ and $\mathbf{m}_{\mathfrak{c}}$.

Then, $\exp \left(-\mathbf{h} \cdot \mathbf{u}_{i} / k_{B} T\right)$ can be linearized, and

$$
k T_{z}=6 J Z^{-1} \int d \Omega u_{z}^{2} e^{-\varepsilon_{A}(\mathbf{u})},
$$

where

$$
Z=\int d \Omega e^{-\varepsilon_{A}(\mathbf{u})},
$$

and $\int d \Omega$ is an integral over all directions of $\mathbf{u}$. Similar equations obtain for the paramagnetic- $x y$-canted phase boundary. For other phase boundaries, linearization cannot be carried out for all spin components, whence slightly more complicated systems of equations ensue. We have solved the equations for the phase boundaries numerically. The solutions for $\varepsilon_{d}=0$ and $C=4 J<0$ are shown in Fig. 10.

It is interesting to compare the ratio $T_{z} / T_{x y}$ between the two relevant temperatures for the SR transition. Some results we have obtained from MC simulations and from the mean field equations above are shown in Fig. 11. How little the results seem to depend on whether SR is brought about purely by dipolar interactions $(J=0)$ or solely by exchange interactions $\left(\varepsilon_{d}=0\right)$ is noteworthy. This may be related to the fact that SR is completely independent of $J$ in the ground state (see Sec. II). How close the mean field and MC data points lie over most of the $0<D / C<1$ range in Fig. 10 is also intriguing. 


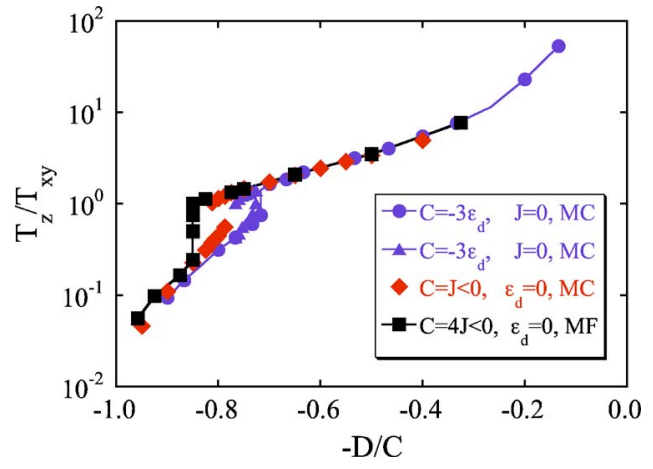

FIG. 11. (Color online) Temperature ratio $T_{x y} / T_{z}$ vs $-D / C$, for the shown values of $\varepsilon_{d}, J$, and $C$ in cubic-shaped systems. "MF" stands for mean field theory results. $-\boldsymbol{\Delta}$ and $\boldsymbol{\Delta}$ stand for $8 \times 8 \times 8$ and $16 \times 16 \times 16$ spin systems, respectively. Data points from MC simulations are for $8 \times 8 \times 8$ spins. Lines are guides to the eye.

\section{E. Reverse SR}

The rather abrupt variation of $T_{z} / T_{x y}$ near $D / C=0.8$ (see Figs. 8 and 11) is worth exploring.

Consider first $J=0$. The behavior that obtains as a function of $T$ for $D=-0.747 C$, and, somewhat irrelevantly, $C$ $=-3 \varepsilon_{d}$, is illustrated in Figs. 12(a) and 12(b). Note that $\left\langle\left|m_{\mathfrak{c}}^{z}\right|\right\rangle$ seems to approach zero in the $0.35 \leqq T \leqq 0.55 \varepsilon_{d} / k_{B}$ range as system size increases. Thus, four phases are visited as $T$ varies in Fig. 12(a): the paramagnetic phase at $0.7 \varepsilon_{d} / k_{B} \lesssim T$, the $z$-collinear phase at $0.55 \lesssim T \lesssim 0.7 \varepsilon_{d} / k_{B}$, the $x y$-canted phase at $0.35 \lesssim T \lesssim 0.55 \varepsilon_{d} / k_{B}$, and the SR phase, at $T \lesssim 0.35 \varepsilon_{d} / k_{B}$. We refer to the SR that takes place within the SR phase,

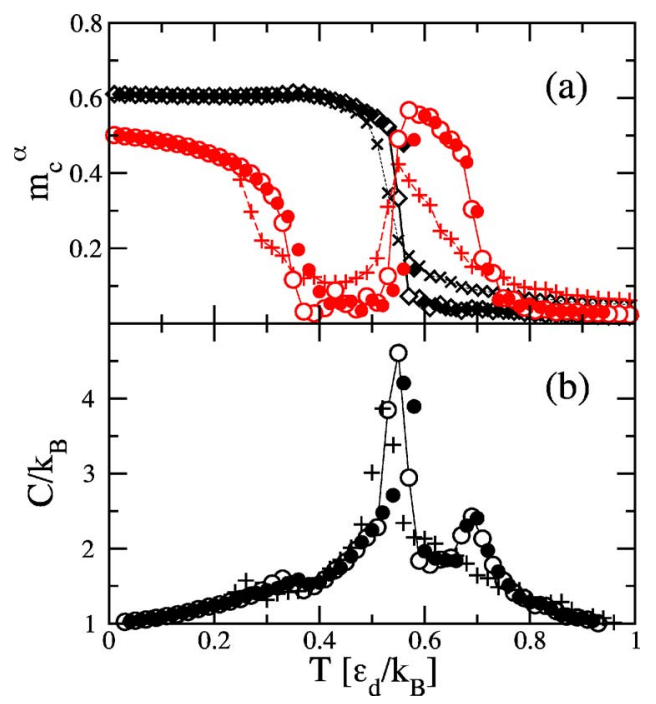

FIG. 12. (Color online) (a) $m_{\mathfrak{c}}^{\alpha}$, for $\alpha=y, z$ vs $T$ for systems of $L \times L \times L$ dipoles with $J=0, C=-3 \varepsilon_{d}$ and $D=0.747 C$. Data points for $m_{\mathfrak{c}}^{x}$ would fall right on top of data points for $m_{\mathfrak{c}}^{y}$ and are not shown. Open (closed) symbols stand for data points from MC simulations in which $T$ was lowered (raised), starting from $T=\varepsilon_{d}(T$ $\left.=0.01 \varepsilon_{d}\right)$. Circles and rhombi stand for $\alpha=z$ and $x$, respectively, both for $L=16$. $+(\times)$ stands for $\alpha=z(\alpha=y)$ for systems with $L$ $=8$. All data points come from averages of $\left|m_{\mathfrak{s}}^{\alpha}\right|$ over some $10^{5} \mathrm{MC}$ sweeps. (b) Same as in (a) but for $C / k_{B}$ vs $T$.

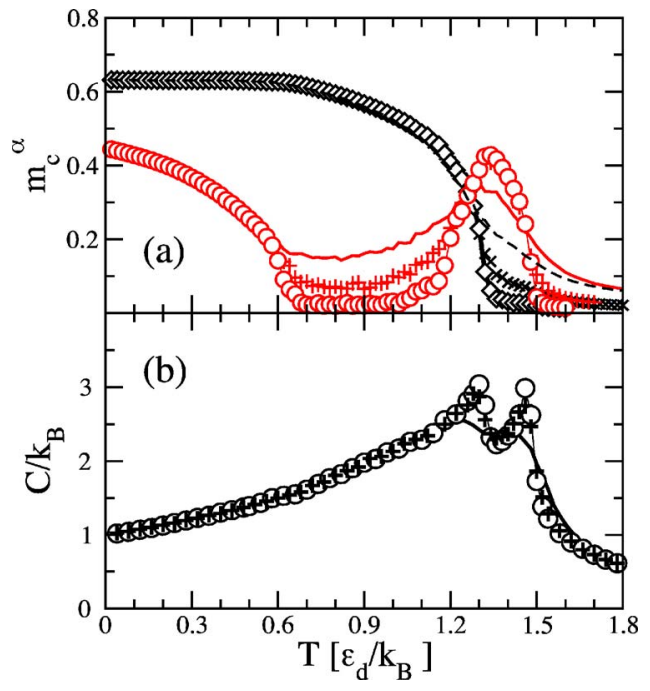

FIG. 13. (Color online) (a) $m_{\mathfrak{s}}^{\alpha}$ for $\alpha=y, z$ vs temperature for systems of $L \times L \times L$ spins with $\varepsilon_{d}=0, C=4 J<0$, and $D=0.8 C$. Data points for $m_{\mathfrak{s}}^{x}$ fall on top of the data points for $m_{\mathfrak{s}}^{y}$ and are therefore not shown. $m_{\mathfrak{s}}^{y}$ is represented by (red online) $\bigcirc$, + , and continuous line for $L=32,16$, and 8 , respectively. $m_{\mathfrak{s}}^{z}$ is represented by (black online), $\diamond, \times$, and dashed line for $L=32,16$, and 8 , respectively. All data points come from averages of $\left|m_{\mathfrak{s}}^{\alpha}\right|$ over 1.8 $\times 10^{5}$ MC sweeps. (b) Same as in (a) but for $C / k_{B}$ vs $T$.

where $T \leqq 0.35 \varepsilon_{d} / k_{B}$ in Fig. 12(a), as a reverse SR, since spin orientations then vary opposite to the way they vary at the higher transition (near $T=0.55 \varepsilon_{d} / k_{B}$ ).

The rather abrupt change in $m_{\mathfrak{c}}^{y}$ and in $m_{\mathfrak{c}}^{z}$ near $T=0.7$ is in accordance with a first-order transition, as in Landau's theory. ${ }^{23}$ (This is so in Landau's theory because neither symmetry group in either of the two phases is a subgroup of the other one. Note that the opposite condition obtains at the other two transition points; hence continuous transitions are predicted therein.)

Reverse SR does not seem to be an isolated phenomenon. It also obtains for other values of $D$, in the range 0.72 $\leq D / C \leqq 0.77$, and for nonzero values of the exchange constant. This is illustrated in Figs. 13(a) and 13(b), for $\varepsilon_{d}=0$, $D / C=0.8$, and, less importantly, $C=4 J$. From plots similar to the one shown in Figs. 12(a) and 12(b), we have obtained for various values of $C / D$ the phase diagram shown in Fig. 14.

\section{CONCLUSIONS}

We have studied the effect of a fourfold anisotropy on the magnetic phases of systems of classical magnetic dipoles on simple cubic lattices with dipolar and antiferromagnetic exchange interactions. For negative anisotropy constants $D$ and $C$, and for $T=0$, we find canted and collinear spin configurations, as shown in Fig. 1 , if $-1.34 \varepsilon_{d} \lesssim J \leqslant 0$ and $J \lesssim$ $-1.34 \varepsilon_{d}$, respectively. An interesting temperature-driven transition between two ordered phases is also reported (see Fig. 4).

We have studied in some depth the spin reorientation phase. Its broken symmetries are exhibited in detail. In our model, a thermally driven continuous SR transition occurs if, 


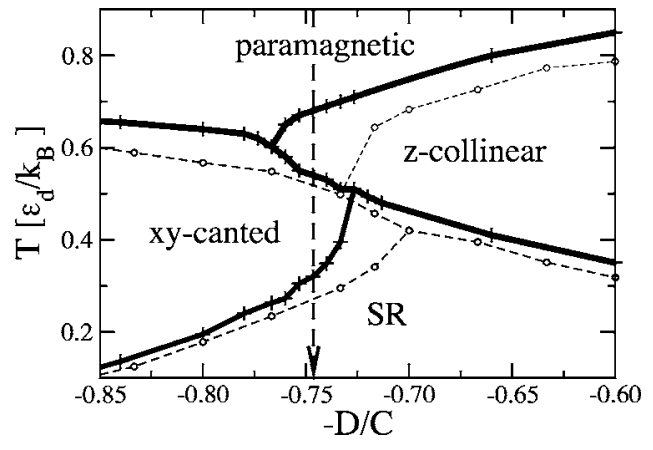

FIG. 14. Magnetic phase boundaries for systems of $L \times L \times L$ spins in which $J=0$ and $C=-3 \varepsilon_{d}$. The full (dashed) line $+(\bigcirc)$ is for $L=16(L=8)$. The vertical dashed line with an arrow is for the cooling path from which the data points in Fig. 12 follow.

in the ground state, spins are tilted away from the crystalline axes. We show, by mean field theory and by MC simulations, that, upon cooling below the paramagnetic phase, collinear (along the easy magnetization axis) spin configurations obtain if $D<0$ and $0 \leqq D / C \leqq 0.8$, and spin reorientation (towards the perpendicular plane) takes place at lower temperatures. On the other hand, if $D<0$ and $0.8 \leqq D / C<1$, spins point perpendicularly to the easy magnetization axis below the paramagnetic phase, and a spin reorientation towards the easy magnetization axis takes place below a lower transition temperature.

Intriguingly, the ratio between the two relevant transition temperatures, $T_{z} / T_{x y}$ (see Fig. 11), does not seem to depend, over most of the $0<D / C<1$ range, on the strength of the exchange interaction, on $C$, or on whether the two temperatures follow from $\mathrm{MC}$ simulations or from mean field thoery.

If $D<0$ and $D / C \sim 0.8$, then (see Figs. 12-14), independent of the strength of the exchange interaction, a complete spin reorientation from the easy magnetization axis into the perpendicular plane first takes place upon cooling below the paramagnetic phase, followed at lower temperature by a reverse spin reorientation, in the SR phase, towards the easy magnetization axis.

\section{ACKNOWLEDGMENTS}

Financial support from Grant No. BFM2003-03919/FISI, from the Ministerio de Ciencia y Tecnología of Spain, is gratefully acknowledged.
*E-mail address: Jefe@Unizar.Es; URL:http://Pipe.Unizar.Es $\sim$ jff

†E-mail address: jjalonso@Uma.Es

${ }^{1}$ G. Ahlers, A. Kornblit, and H. J. Guggenheim, Phys. Rev. Lett. 34, 1227 (1975); G. Mennenga, L. J. de Jongh, and W. J. Huiskamp, J. Magn. Magn. Mater. 44, 59 (1984); M. R. Roser and L. R. Corruccini, Phys. Rev. Lett. 65, 1064 (1990); S. J. White, M. R. Roser, J. Xu, J. T. van der Noordaa, and L. R. Corruccini, ibid. 71, 3553 (1993); D. Bitko, T. F. Rosenbaum, and G. Aeppli, ibid. 77, 940 (1996).

${ }^{2}$ See, for instance, D. Gatteschi and R. Sessoli, in Magnetism: Molecules to Materials, edited by J. S. Miller and M. Drillon (Wiley-VCH, Weinheim, 2002), Vol. III, Chap. 3.

${ }^{3}$ Quantum Tunneling of the Magnetisation, edited by L. Gunther and B. Barbara (Kluwer, Dordrecht, 1995).

${ }^{4}$ E. M. Chudnovsky and J. Tejada, Macroscopic Quantum Tunneling of the Magnetic Moment (Cambridge University Press, Cambridge, 1998).

${ }^{5}$ J. F. Fernández, in Modeling of Complex Systems, edited by P. L. Garrido and J. Marro (American Institute of Physics, Melville, NY, 2003), pp. 242-247.

${ }^{6}$ D. Gatteschi, R. Sessoli, and J. Villain, Molecular Nanomagnets (Oxford University Press, Oxford, 2006).

${ }^{7}$ Exchange interactions can nevertheless be appreciable in some cases; see, for instance, W. Wernsdorfer, N. Aliaga-Alcalde, D. N. Hendrickson, and G. Christou, Nature (London) 416, 406 (2002); R. Tiron, W. Wernsdorfer, N. Aliaga-Alcalde, and G. Christou, Phys. Rev. B 68, 140407(R) (2003); S. Hill, R. S. Edwards, N. Aliaga-Alcalde, and G. Christou, Science 302, 1015 (2003); W. Wernsdorfer, S. Bhaduri, A. Vinslava, and G. Christou (unpublished).

${ }^{8}$ M. Affronte, J. C. Lasjaunias, W. Wernsdorfer, R. Sessoli, D. Gatteschi, S. L. Heath, A. Fort, and A. Rettori, Phys. Rev. B 66,
064408 (2002).

${ }^{9}$ A. Morello, F. L. Mettes, F. Luis, J. F. Fernández, J. Krzystek, G. Aromí, G. Christou, and L. J. de Jongh, Phys. Rev. Lett. 90, 017206 (2003).

${ }^{10}$ A. Morello, F. L. Mettes, O. N. Bakharev, H. B. Brom, L. J. de Jongh, F. Luis, J. F. Fernández, and G. Aromí, Phys. Rev. B (to be published).

${ }^{11}$ M. Evangelisti, F. Luis, F. L. Mettes, N. Aliaga, G. Aromí, J. J. Alonso, G. Christou, and L. J. de Jongh, Phys. Rev. Lett. 93, 117202 (2004).

${ }^{12}$ F. Luis, J. Campo, J. Gómez, G. J. McIntyre, J. Luzón, and D. Ruiz-Molina, Phys. Rev. Lett. 95, 227202 (2005).

${ }^{13}$ J. F. Fernández, Phys. Rev. B 66, 064423 (2002).

${ }^{14}$ J. Luttinger and L. Tisza, Phys. Rev. 72, 257 (1947); see also Th. Niemeijer and H. W. J. Blötte, Physica (Amsterdam) 67, 125 (1973); S. K. Misra and J. Felsteiner, Phys. Rev. B 15, 4309 (1977).

15 J. F. Fernández and J. J. Alonso, Phys. Rev. B 62, 53 (2000); 65, 189901(E) (2002); for a treatment of magnetic order in $\mathrm{Fe}_{8}$ crystals, see also X. Martínez-Hidalgo, E. M. Chudnovsky, and A. Aharony, Europhys. Lett. 55, 273 (2001).

${ }^{16}$ E. M. Gyorgy, J. P. Remeika, and F. B. Hagedorn, J. Appl. Phys. 39, 1369 (1968).

${ }^{17}$ For a nice early view of the subject, see W. P. White, J. Appl. Phys. 40, 1061 (1969).

${ }^{18}$ See for instance, R. Allenspach and A. Bischof, Phys. Rev. Lett. 69, 3385 (1992); A. Berger and H. Hopster, ibid. 76, 519 (1996).

${ }^{19}$ H. P. Oepen, M. Speckmann, Y. Millev, and J. Kirschner, Phys. Rev. B 55, 2752 (1997).

${ }^{20}$ For treatments of a first-order SR transition, assuming no higher anisotropies beyond the uniaxial one, see: (i) for renormalization 
group calculations, D. Pescia and V. L. Pokrovsky, Phys. Rev. Lett. 65, 2599 (1990); 70, 1185 (1993); A. P. Levanyuk and N. García, ibid. 70, 1184 (1993); (ii) for MC simulations, C. Santamaria and H. T. Diep, J. Magn. Magn. Mater. 212, 23 (2000); (iii) for a mean field calculation (in which $C=0$ ), L. Udvardiyz, L. Szunyoghyz, A. Vernesz, and P. Weinbergerz, Philos. Mag. B 81, 613 (2001).

${ }^{21}$ For reviews of effects of dipole-dipole interactions on the phases of thin films, see K. De'Bell, A. B. MacIsaac, and J. P. Whitehead, Rev. Mod. Phys. 72, 225 (2000); J. J. Weis, J. Phys.: Condens. Matter 15, 1471 (2003).

${ }^{22}$ A. M. Abu-Labdeh, J. P. Whitehead, K. De'Bell, and A. B. MacIsaac, Phys. Rev. B 65, 024434 (2002); 70, 144416 (2004).

${ }^{23}$ L. D. Landau and E. M. Lifshitz, Electrodynamics of Continuous Media, 2nd ed. (Pergamon, Oxford, 2004), pp. 159-162. The "angular phase" is how Landau and Lifshitz refer to the SR phase.

${ }^{24}$ H. Horner and C. M. Varma, Phys. Rev. Lett. 20, 845 (1968); F. B. Hagedorn and E. M. Gyorgy, Phys. Rev. 174, 540 (1968).

${ }^{25}$ To see why hyperfine interactions have no thermal equilibrium effects on classical electronic spins, consider adding a hyperfine interaction $\mathcal{H}_{h p}$ to the Hamiltonian. We assume all other nuclear spin interactions are negligible. Let $\mathcal{H}_{h p}=-A \Sigma_{i} \mathbf{S}_{i} \cdot \mathbf{I}_{i}$, where $\mathbf{I}_{i}$ is the nuclear spin that goes with the $i$ site. Then, $\operatorname{Tr} \exp [-(\mathcal{H}$ $\left.\left.+\mathcal{H}_{h p}\right) / k_{B} T\right] \rightarrow \operatorname{Tr}_{\{S\}} \exp \left[-(\mathcal{H}) / k_{B} T\right] \operatorname{Tr}_{\{S, I\}} \exp \left[-\left(\mathcal{H}_{h p}\right) / k_{B} T\right] \quad$ if all $\mathbf{S}_{i}$ are classical. It follows that hyperfine interactions have no effect, since the trace over all nuclear spins $\{I\}$ is clearly independent of the directions of $\{S\}$.

${ }^{26}$ See Bitko, et al., in Ref. 1; P. B. Chakraborty, P. Henelius, H. Kjonsberg, A. W. Sandvi, and S. M. Girvi, Phys. Rev. B 70, 144411 (2004).

${ }^{27}$ N. A. Metropolis, A. W. Rosenbluth, M. N. Rosenbluth, A. H. Teller, and E. Teller, J. Chem. Phys. 21, 1087 (1953).

${ }^{28}$ R. B. Griffiths, Phys. Rev. 176, 655 (1968).

${ }^{29}$ Invariance of $E_{d}$ with respect to $\phi$ rotations in the canted state has been pointed out for the special case of $\theta=\pi / 2$, by K. De'Bell, A. B. MacIsaac, I. N. Booth, and J. P. Whitehead, Phys. Rev. B 55, 15108 (1997).

${ }^{30}$ A. B. MacIsaac, J. P. Whitehead, K. De'Bell, and P. H. Poole, Phys. Rev. Lett. 77, 739 (1996).

${ }^{31}$ A thermally induced quadrupolar anisotropy that comes from dipolar interactions has been predicted for films in K. De'Bell, A. B. MacIsaac, I. N. Booth, and J. P. Whitehead, Phys. Rev. B 55, 15108 (1997); J.-C. S. Lévy, ibid. 63, 104409 (2001). 